\title{
Realistische Inwertsetzung statt Abgeltungen von Kosten
}

\author{
Die Monetarisierung von Ökosystemleistungen \\ gewinnt nicht zuletzt vor dem Hintergrund der \\ Debatte um Biodiversität an Bedeutung. Mone- \\ tarisierungsstudien für den Wald sollen am Bei- \\ spiel der Schweiz den derzeitigen Forschungstand \\ aufzeigen. Doch erfahren Ökosystemleistungen \\ des Waldes den notwendigen Schutz und und wie \\ werden sie abgegolten? \\ Von Karin Ingold und Tiana Moser
}

I. n gesellschaftlichen und politischen Auseinandersetzungen ist ein zunehmender Trend hin zu einer Ökonomisierung beobachtbar. Folglich verdrängen Gesichtspunkte des Marktes marktfremde Gesichtspunkte zunehmend aus dem Gesellschaftsleben (Grove-White 1997; Pelizzari 2001; Thielmann 2004a; 2004b). Eine Ausprägung der Ökonomisierung stellt die vermehrte Bezugnahme auf monetäre Einheiten in der Beurteilung von Policy Problemen und deren Lösungen dar.

Dies zeigt sich an verschiedenen Studien zur Bewertung gesamtgesellschaftlich anfallender Kosten und Leistungen. Monetarisierungen versuchen einen verzerrten Wert zu korrigieren oder jene Aspekte in Geldeinheiten auszudrücken, welche nicht bereits einen Preis über den Markt erhalten. Im Umweltbereich ist dies von zentraler Bedeutung, da es sich meist um öffentliche Güter handelt.

\section{Inwertsetzung von Ökosystemleistungen}

Dieser Trend zeigt sich besonders deutlich im Bereich Wald. Natürliche Ressourcen wie der Wald werden nicht nur genutzt, sondern weisen auch gesamtgesellschaftliche Leistungen auf. Beispiele hierfür sind der Erholungswert oder die Biodiversität des Waldes.

Im globalen Diskurs über Mensch-Umwelt-Beziehungen hat sich für solche Leistungen der Begriff der Ecosystem Services etabliert (Alcamo 2003): „Ecosystem services are the benefits people obtain from ecosystems. " Gemäß der funktionalen Klassierung von Ecosystem Services, oder Ökosystemleistungen, können die folgenden Typen von Waldleistungen unterschieden werden (Alcamo 2003):

- Provisioning services: Holz, Wildtiere, Pilze, genetische Ressourcen etc.

- Regulating services: Luftreinigung, Klimaregulierung, Schutz vor Naturgefahren etc.
- Cultural services: kulturelle Vielfalt, ästhetische Vielfalt, Erholung etc.

- Supporting services: Photosynthese, Nährstoffkreislauf, Wasserkreislauf etc.

Waldleistungen können zudem nach dem Kriterium der Marktfähigkeit kategorisiert werden. Während beispielsweise Holzprodukte auf Märkten gehandelt werden, zählen die kulturelle oder ästhetische Vielfalt zu den nicht marktfähigen Waldleistungen. Diese Leistungen sind aufgrund gesellschaftlicher und ökonomischer Entwicklungen im Verlauf der letzten Jahre vermehrt zum Gegenstand forstökonomischer und -politischer Analysen geworden (siehe beispielsweise Linddal 1994; Bishop 1998; Langner 1998; Janse/Ottitsch 2005; Seeland/Staniszewski 2006). Solche Leistungen tauchen in der forstlichen Literatur auch unter dem Begriff der Wohlfahrtsfunktionen oder Non Timber Forest Goods and Services auf.

Abgesehen von den eigentlichen Bewertungen interessiert zudem, inwiefern Ökosystemleistungen in Wert gesetzt werden und dadurch zum Beispiel versucht wird, die Leistung monetär abzugelten. Im vorliegenden Artikel gehen wir der Frage nach, welche Waldleistungen in der Schweiz nach welchen Methoden bewertet wurden und zu welchen Werten einzelne Studien gekommen sind. Zudem interessiert uns, ob basierend auf solchen Studien oder unabhängig von diesen Waldleistungen bereits in Wert gesetzt beziehungsweise abgegolten werden.

\section{Monetarisierungsstudien zu Nichtholzleistungen}

Monetarisierungsstudien aus dem deutschsprachigen Raum systematisch zu sammeln und zu vergleichen erweist sich aufgrund der sehr heterogenen Forschungsziele und der unterschiedlich angewandten Methoden als schwierig (Elsasser/Meyerhofer 2007). Am häufigsten wurden sowohl in der Schweiz wie auch in den deutschsprachigen Nachbarländern Studien zum Wert der Erholungsfunktion des Waldes erstellt. Diese Bewertungen wurden primär mittels der Reisekostenmethode und der kontingenten Bewertungsmethode erstellt (Moser 2008). Die Werte weisen dabei eine enorme Spannweite von 3,5 bis 65.000 Schweizer Franken pro Hektar und Jahr auf. Andere Leistungen als der Erholungswert wurden bisher kaum monetarisiert. Im Falle der Schutzwirkung des Waldes etwa fand keine systematische Monetarisierung statt, obwohl sie in Berggebieten eine wichtige Stellung einnimmt.

In einem ersten Schritt haben wir einen Überblick über die Verankerung von Schweizer Ökosystemleistungen auf der $\rightarrow$ 
Stufe von Verwaltungsprogrammen und teilweise von Politikkonzepten erstellt. Dieser Überblick wurde basierend auf einer Dokumentenanalyse von rechtlichen Grundlagen erstellt. Daraus resultierte, dass die Zahlungen der einzelnen Sektorpolitiken wie Wald, Landwirtschaft, Wasser sowie Natur- und Landschaftsschutz den Leistungen nicht eindeutig zuzuordnen sind. Besonders deutlich kommt dies bei der Landwirtschaftspolitik zum Ausdruck, denn hier erfolgen meist Zahlungen für die gleichzeitige Bereitstellung von verschiedenen Leistungen wie provisioning (Biodiversität), cultural (Landschaftsschutz, Kulturelles Erbe) und supporting services (Erhaltung der Böden). Im Bereich Wald dominieren hingegen Zahlungen für provisioning (Biodiversität) und regulating services (Schutzwald).

In einem zweiten Schritt wurde das Augenmerk auf konkrete Inwertsetzungen von Ökosystemleistungen gelegt. Die Inwertsetzungen der Leistungen der Forstwirtschaft gehören zur Gruppe der „Payments for Ecosystem Services“, kurz PES. Nach Wunder sind PES ,eine freiwillige Transaktion bei der eine genau definierte Umweltleistung von mindestens einem Käufer gekauft und von mindestens einem Produzenten verkauft wird, unter der Voraussetzung, dass der Produzent die Bereitstellung der Umweltleistung sicherstellt“ (Wunder 2005). Darauf basierend lassen sich folgende Leitfragen für die Untersuchung der Inwertsetzung von Waldfunktionen ableiten:

I Welche Leistungen werden erbracht beziehungsweise abgegolten?

I Wer sind mögliche und wahrscheinliche Leistungserbringer?

- Wer sind mögliche und wahrscheinliche Abnehmer?

I Was sind mögliche zentrale formale Kriterien für die Teilnahme am Programm?

- Auf welcher Basis, zum Beispiel Bewertungen von Leistungen und Aufwendungen oder Aushandlungen, basieren die Zahlungen beziehungsweise wie wird der Preis der Leistung festgelegt?

- Wie wird kontrolliert, ob die Regeln des Programms eingehalten werden?

I Gibt es Sanktionen bei Verstößen gegen die Regeln?

Neben der genauen Definition der zu erbringenden Leistung ist auch die Identifikation des Leistungserbringers und der potenziellen Käufer zentral. Hier ist insbesondere interessant, ob die Transaktion zwischen privaten Akteuren oder im Rahmen eines staatlichen Förderprogramms stattfindet. Die fünfte Leitfrage bezieht sich auf die Höhe der Zahlung. Basiert die Zahlung etwa auf dem Nutzen für den Käufer oder eher auf den Kosten der Bereitstellung? Die beiden letzten Leitfragen konzentrieren sich auf Kontrollmaßnahmen und mögliche Sanktionen (Moser 2008). Diese definierten Leitfragen werden nachfolgend auf vier ausgewählte Beispiele angewandt. Die Resultate basieren auf einer Dokumentenanalyse und Experteninterviews.

\section{Analyse von Fallbeispielen}

Für die Analyse der Inwertsetzung wurden vier Fälle mit jeweils unterschiedlichen Ökosystemleistungen ausgewählt. Zur
Anwendung der oben genannten Leitfragen wurden eine Literaturrecherche sowie einzelne Expertengespräche durchgeführt. Die Beispiele zeigen auf, dass eine Inwertsetzung in der Schweiz bereits verschiedentlich erfolgt. Ein Fall richtet sich auf pauschale Inwertsetzungen im Rahmen von Leistungsvereinbarungen im Kanton Basel-Land. Das heißt, es werden verschiedene Ökosystemleistungen gemeinsam abgegolten. Ein zweiter Fall konzentriert sich auf die Inwertsetzung von Wasserreinigungs- und Rückhaltefunktionen im Kanton Solothurn. Im dritten und vierten Fall wird die Inwertsetzung von Biodiversitätsfunktionen im Kanton Graubünden beziehungsweise der Erholungsfunktion im Kanton Fribourg analysiert.

\section{Leistungen und Leistungserbringer}

Die Differenzierung der einzelnen Leistungen ist oft schwierig und deren Erfassung mit enormem Aufwand verbunden. Es bietet sich deshalb eine pauschale Abgeltung für verschiedene Leistungen an. Dieser Grundsatz wird im Waldgesetz des Kantons Basel-Land festgehalten. Im Forstkreis Sissach wurde darauf basierend eine Leistungsvereinbarung zwischen Forstbetrieben und den Einwohnergemeinden als Abnehmer getroffen. Der Leistungskatalog umfasst dabei Maßnahmen zur Erhaltung und Förderung der Artenvielfalt, der ästhetisch schönen Waldbilder oder den Trinkwasserschutz. Die Abgeltungsbeträge belaufen sich dabei auf zwischen sechs und 18 Schweizer Franken pro Hektar und Jahr. Die Unterschiede basieren dabei auf Aufwandberechnungen.

Im Einzugsgebiet einer Grundwasserfassung ist die Waldbewirtschaftung und -pflege wesentlich verantwortlich für die Qualität des Grundwassers. Basierend auf der nationalen Gesetzgebung hat der Kanton Solothurn Richtlinien für die Entschädigung an die Forstbetriebe durch die Abnehmer, meist Einwohnergemeinden, verfasst. Der Kanton Solothurn hat eine Reihe von Auflagen für drei Grundwasserschutzzonen definiert. Die Auflagen werden mit zunehmender Schutzstufe umfangreicher. Maßnahmen reichen von einem Verzicht auf die Verwendung chemischer Pflanzenbehandlungsmittel und die Lagerung von behandeltem Holz, über das Verbot Maschinen über Nacht im Wald zu lassen bis zu einem umfassenden Verzicht auf eine Waldnutzung. Die Beträge variieren je nach Auflagen zwischen 30 und 130 Schweizer Franken pro Hektar und Jahr.

Die Wälder sollen einen wesentlichen Beitrag zur Biodiversität der Schweiz leisten (BUWAL 2002). Basierend auf dem nationalen Waldgesetz leistet der Bund bis zu 50 Prozent der Kosten als Finanzhilfe an Schutz- und Unterhaltsmaßnahmen (Art. 38 Abs. 3 WAG). Neben dem Bund, den Kantonen und den Gemeinden beteiligen sich zudem auch private Verbände an Abgeltungen. Ein Beispiel für die Abgeltung von Biodiversitätsfunktionen ist das Waldreservat Val Cama und Val Leggia im Kanton Graubünden. Mit der Einrichtung des Waldreservates und den entsprechenden Abgeltungen an die Gemeinden werden unter anderen folgende Ziele verfolgt: Schutz von seltenen Pflanzen und Tieren, Erwerb von waldbaulichen Erkenntnissen 
für die optimale Bewirtschaftung der Schutzwälder sowie Erkenntnisse über die langfristige natürliche Entwicklung des Waldes ohne menschliche Eingriffe. Konkret verzichten die Bürgergemeinden auf jegliche Haupt- und Nebennutzungen. Für das gesamte Gebiet von 1500 Hektar wird an die Gemeinden für die Vertragsdauer von 50 Jahren ein Betrag von 370.000 Schweizer Franken plus 99.000 Schweizer Franken Forstdepositum gutgeschrieben. Dieser Betrag liegt deutlich unter dem kantonalen Eckwert von 20 Schweizer Franken pro Hektar und Jahr, welcher für die Abgeltung zur Einrichtung von Naturwaldreservaten vorgesehen ist.

Die Erholungsfunktion ist Teil der Wohlfahrtsfunktionen des Waldes, wie sie im Zweckartikel des nationalen Walgesetzes festgehalten werden (Art. $1 \mathrm{WaG}$ ). Zur Sicherstellung der Erholungsfunktion werden meist von den Waldbewirtschaftern zusätzliche Leistungen erbracht, welche eine Nutzung zur Erholung überhaupt zulassen. Dazu zählen etwa die Instandhaltung eines Wegnetzes, die Gewährleistung der Sicherheit und die Bereitstellung von Grill- oder Picknickplätzen.

Im Kanton Fribourg wurde 2006 ein Projekt zur Entschädigung der Erholungsfunktion in öffentlichen Wäldern gestartet. Zur Sicherstellung der Erholungsfunktion entschädigt der Kanton versuchsmäßig die Bewirtschaftungskosten, welche den Forstbetrieben dadurch anfallen. Diese Leistungen umfassen unter anderem zusätzliche Kosten zur Schaffung oder Verjüngung von Beständen mit Erholungsfunktion und defizitärer Holzernte aus Sicherheitsgründen (Kantonales Amt für Wald, Wild und Fischerei Fribourg 2006). Nicht Bestandteil dieser Leistungen sind hingegen der Bau und Unterhalt von Erholungseinrichtungen wie Bänke, Informationstafeln, Lehrpfade sowie die Beseitigung von Abfall. Die Abgeltungspauschalen betragen in Abhängigkeit von Intensität der Erholungsfunktion zwischen 200 und 1000 Schweizer Franken pro Hektar und Jahr.

\section{Festlegung des Preises}

Die Höhe der Inwertsetzung basiert in drei der vier Fälle primär auf den Kosten. Die pauschalen Abgeltungen im Kanton Basel-Land werden basierend auf den tatsächlich anfallenden Kosten, den Betriebszahlen und einer Studie in der Region zu den Kosten der Erholungsfunktion berechnet (Baur/Bilecen 2003). Auch im Falle der Inwertsetzung der Wasserreinigungsund Rückhaltefunktion im Kanton Solothurn wird der Betrag basierend auf den Kosten festgelegt. Zusätzlich wird jedoch der Holzerlös mit einberechnet, sodass für die Inwertsetzung schließlich die Differenz maßgebend ist. Die Inwertsetzung der Erholungsfunktion im Kanton Fribourg basiert im Vergleich zu den anderen beiden Beispielen auf den reinen Kosten. Die Inwertsetzung der Biodiversitätsfunktion im Kanton Graubünden hingegen basiert als einziges Beispiel nicht auf den Kosten, sondern ist ein Resultat von Verhandlungen basierend auf der realen Zahlbarkeit von Abgeltungen.

Die Monitoring- und Sanktionsmaßnahmen sind kaum ausgebildet. Die Überprüfung der Erfüllung der Leistungen im Bei-

\section{"Neben der Holzproduktion wird als Leistung des Waldes zumeist nur der Wert der Erholungsfunktion berechnet."}

spiel der pauschalen Inwertsetzung im Kanton Basel-Land erfolgt über die Betriebsabrechnung der Forstbetriebe und über die soziale Kontrolle, das heißt durch die Waldbesucher. Die einzig mögliche Sanktionsmaßnahme stellt die Vertragskündigung dar. Das Monitoring erfolgt im Falle der Wasserreinigungsleistungen durch die Empfänger der Leistungen und somit die Einwohnergemeinde beziehungsweise den verantwortlichen Brunnenmeister. Sanktionsmechanismen existieren keine. Im Rahmen der Inwertsetzung der Biodiversitätsfunktion im Kanton Graubünden existiert kein Monitoringprogramm, hingegen würde als Sanktionsmechanismus die Vertragskündigung zur Anwendung kommen. In diesem Fall müssten die Gemeinden die Beträge zurückbezahlen. Im Falle der Inwertsetzung der Erholungsfunktion im Kanton Fribourg überprüfen die zuständigen Revier- oder Kreisförster die Erbringung der Leistungen. Bei einer Nichterbringung würde es zu Kürzungen der Beträge kommen.

\section{Schlussfolgerungen}

Zusammenfassend kann festgehalten werden, dass die Inwertsetzung basierend auf den realen Kosten oder dem berechneten Wert von Waldleistungen in der Schweiz noch in den Anfängen begriffen ist. Einerseits sieht sich die methodische Basis zur Bewertung von Umwelt- beziehungsweise Waldleistungen nach wie vor mit großen Herausforderungen konfrontiert. Dies zeigen die unterschiedlichen Resultate der Studien deutlich. Eine Typologie von vier Leistungen findet jedoch bereits eine breite Anwendung. Andererseits weisen Bewertungen zumindest in der Schweiz kaum empirische Relevanz auf. Die Auswertung der Studien zeigt, dass zumindest im deutschsprachigen Raum primär die Reisekostenmethode und die kontingente Bewertungsmethode angewandt werden und dies hauptsächlich zur Bewertung der Erholungsfunktion. Aufgrund der Anwendung verschiedener Methoden und Werte ist es aber schwierig, wenn nahezu unmöglich, die Resultate der verschiedenen Studien und somit die Inwertsetzung unterschiedlicher Ökosystemleistungen miteinander zu vergleichen.

Ein erster Ansatz in die Richtung einer systematischen Analyse von Ökosystemleistungen und deren Abgeltung sollen die in dieser Arbeit formulierten Leitfragen bieten. Insgesamt konnten nur wenige Beispiele für Inwertsetzungen identifiziert $\rightarrow$ 
werden. Die Anwendung der Leitfragen auf vier Schweizer Fallstudien hat vor allem gezeigt, dass Zahlungen, falls sie denn erfolgen, beinahe ausschließlich auf Kosten und nicht auf monetären Bewertungen basieren. Es handelt sich somit um Abgeltungen von Kosten und nicht um eine Inwertsetzung basierend auf berechneten monetären Werten von Leistungen. Zudem zeigen die Beispiele, dass die real beobachteten Inwertsetzungen im Vergleich zu den berechneten Beträgen der Wissenschaft relativ niedrig sind. Die dargelegte Thematik wird im Rahmen des Forschungsprojektes „Valorisation of Non Timber Forest Goods and Services: From Research Results to Political Decisions“ vertieft betrachtet werden.

\section{Literatur}

Alcamo, J.: Ecosystems and human well-being: a framework for assessment. Washington 2003.

Bishop, J.T.: The Economics of Non-Timber Forest Benefits: An Overview. International Institute for Environment and Development. London 1998.

Baur, B. / Bilecen, E.: Freizeitaktivitäten im Baselbieter Wald: ökologische Auswirkungen und ökonomische Folgen. Liestal 2003.

BUWAL: Leitsätze einer Waldreservatspolitik in der Schweiz. Bern 2002.

Elsasser, P. / Meyerhofer, J.: A data base on environmental valuation studies in Austria, Germany and Switzerland. 2007. Internet: http://www.bfah.de/bibl/pdf/iii_07_01.xls.

Grove-White, R.: The Environmental "Valuation" Controversy. Observations on its recent history and significance. In: Foster, J. (Hrsg.): Valuing Nature? Ethics, economics and the environment. London, New York 1997.

Janse, G. / Ottitsch, A.: Factors influencing the role of Non-Wood Forest Products and Services. In: Forest Policy and Economics 3/2005, S. 309-319.

Kantonales Amt für Wald, Wild und Fischerei Fribourg: Entschädigung der Erholungsfunktion in öffentlichen Wäldern, die in rationellen Bewirtschaftungseinheiten eingebunden sind. Direktion der Institutionen und der Land- und Forstwirtschaft. Kanton Fribourg 2006.

Langner, L.: Non-wood goods and services of the forests. New York, Geneva 1998.

Linddal, M.: Assessment of non-wood goods and services: a forest policy approach. The Monte Verità Conference on Forest survey designs. Ascona 1994.
Moser, T. / Zabel, A. / Bernath, K. / Baur, P. / Roschewitz, A. / Beck, C. / Zimmermann, W.: Inwertsetzung von Waldwerten und Waldleistungen. Ergänzungsbeitrag zu Cost Aktion E45. Zürich 2008.

Pelizzari, A.: Die Ökonomisierung des Politischen new public management und der neoliberale Angriff auf die öffentlichen Dienste. Konstanz 2001.

Seeland, K. / Staniszewski, P.: Indicators for a European cross-country assessment of non-timber forest products and services. In: Niskanen, A. (Hrsg.): Issues Affecting Enterprise Development in the Forest Sector in Europe. Joennsuu 2006. S. 299-320.

Thielemann, U.: Integrative Wirtschaftsethik als Reflexionsbemühungen im Zeitalter der Ökonomisierung. In: Mieth, H. / Schumann, O.J. / Ulrich, P. (Hrsg.): Reflexionsfelder integrativer Wirtschaftsethik. Tübingen, Basel $2004 a$.

Thielemann, U.: Transzendentale Ökonomik. Bemerkungen zur Ökonomisierung der Wissenschaften. In: Forschung \& Lehre 7/2004b, S. 358.

Wunder, S.: Payments for environmental services: Some nuts and bolts. $\mathrm{Cl}-$ FOR Occasional Paper No. 42, 2005

\section{AUTORINNEN + KONTAKT}

Dr. Karin Ingold ist Politologin und seit 2008 Forscherin und Dozentin am Institute for environmenta decisions (IED) an der Eidgenössischen Technischen Hochschule (ETH) Zürich.

Tiana Moser ist seit 2005 Forscherin am IED und schreibt seit 2007 an ihrer Dissertation.

Institute for environmental decisions (IED), ETH Zürich, Universitätsstr.22, 8092 Zürich, Schweiz. Tel.: +41446323217,

E-Mail: tiana.moser@env.ethz.ch,kingold@ethz.ch
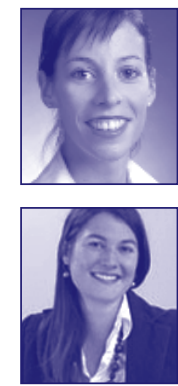

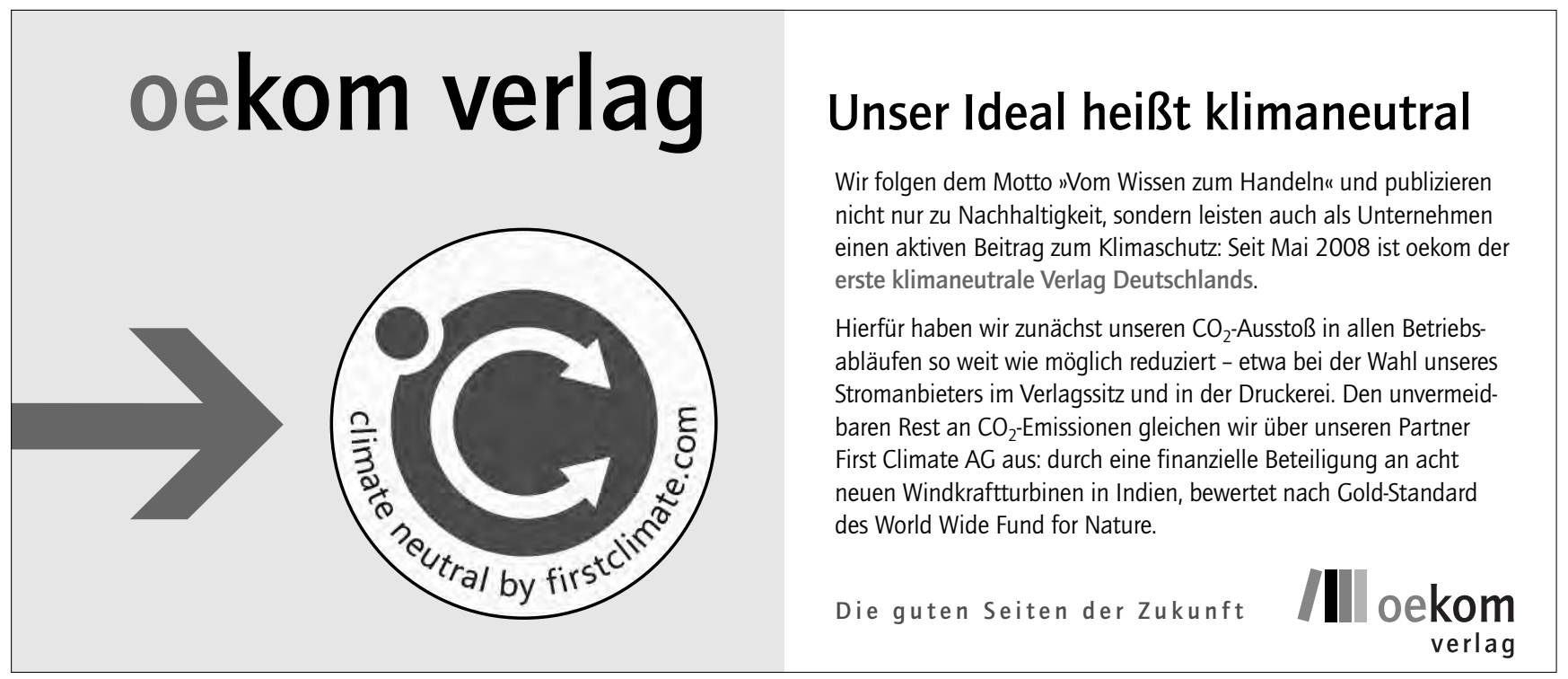


(c) 20I0 Authors; licensee IÖW and oekom verlag. This is an article distributed under the terms of the Creative Commons Attribution Non-Commercial No Derivates License (http://creativecommons.org/licenses/by-nc-nd/3.o/), which permits unrestricted use, distribution, and reproduction in any medium, provided the original work is properly cited. 